\title{
Nicht bei der Heizung sparen!
}

\section{Eine moderne Heizungsanlage kann offenbar Asthmasymptome bei Kindern bessern - dank geringerer Schadstoffkonzentrationen in der Luft und weniger Feuchtigkeit und Schimmelbildung.}

$\mathrm{n}$ vielen Ländern - beispielsweise im Mittelmeerraum oder auch auf der anderen Seite der Weltkugel, z. B. in Ozeanien, - sind nicht alle Haushalte mit einer Zentralheizung ausgestattet, auch wenn durchaus kühle Wintertage vorkommen. Niedrige Temperaturen, Feuchtigkeit und Schimmel sowie die Luftbelastung mit Stäuben (z.B. aus Kaminen) und Stickoxiden können die Atemwege belasten. Deshalb untersuchte eine randomisierte kontrollierte Studie in neuseeländischen Haushalten, ob ein gutes Heizsystem die Symptome von Kindern mit Asthma bronchiale bessern kann. Dazu wurden in Haushalten mit asthmakranken Kindern im Alter von sechs bis zwölf Jahren vor der Wintersaison entsprechende Anlagen mit Wärmepumpe bzw. Holzpelletoder Gaszentralheizungen eingebaut. Der Kontrollgruppe wurde eine entsprechende Heizanlage nach Ende der Studie angeboten.
Durch den Einbau der Zentralheizung stieg die mittlere Temperatur im Wohnraum um $1,1{ }^{\circ} \mathrm{C}$, im Kinderschlafzimmer um $0,57^{\circ} \mathrm{C}$ an. Gleichzeitig wurde signifikant weniger Stickoxid im Vergleich zu den Kontrollhaushalten gemessen. Die Lungenfunktion,

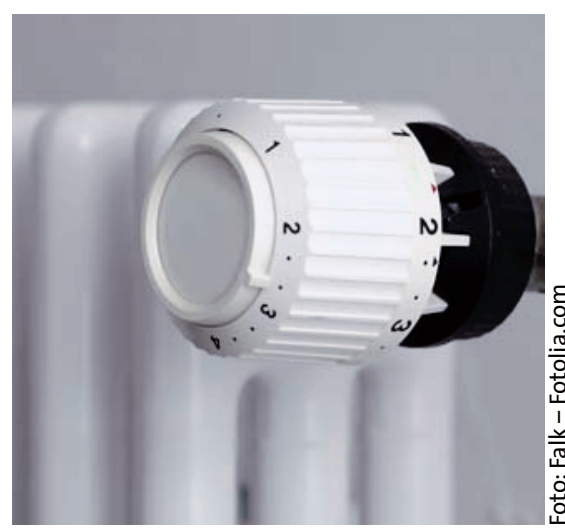

Schlicht aber wahr: Eine funktionierende Zentralheizung kann die Symptome asthmakranker Kinder vermindern.

\section{Schulung schützt vor Krankenhaus}

\section{Patientenschulungen sind heute ein wichtiger Bestandteil im Management chronischer Erkrankungen. Der Nutzen von Schulungsmaßnahmen bei Kinder und Jugendlichen mit Asthma bronchiale wurde jetzt anhand einer Metaanalyse hinterfragt.}

E pidemiologen aus Berkeley, Kalifornien, bezogen in ihre Metaanalyse insgesamt 37 US-amerikanische Studien mit Kindern und Jugendlichen im Alter zwischen zwei und 17 Jahren mit gesicherter Asthmadiagnose ein, in denen der Effekt einer Asthmaschulung untersucht worden war. Als Zielparameter wurden asthmabedingte Krankenhauseinweisungen, Notaufnahmen und Notfallbesuche durch einen Arzt analysiert.

Bei den Studien mit üblicher Versorgung im Vergleich zur Schulungsintervention ergaben sich für die geschul- ten Asthmapatienten eine statistisch signifikante Abnahme der Zahl von Krankenhauseinweisungen und Notfallaufnahmen sowie ein Trend zu einer geringeren Odds Ratio für Notfallaufnahmen. Die Odds Ratio für Krankenhauseinweisungen und die Zahl von Notfallbesuchen durch den Arzt war in beiden Gruppen jedoch ähnlich hoch. Dieses zunächst paradox erscheinende Ergebnis könnte sich mit einer in den Schulungen trainierten Verhaltensänderung bei der Inanspruchnahme medizinischer Hilfe erklären lassen. Ein rasches Aufsuchen der primäre Endpunkt der Studie, verbesserte sich durch den Heizungseinbau allerdings nicht signifikant. Die höhere Raumtemperatur bei gutem Raumklima hatte dennoch deutlich spürbare Effekte: Die Kinder aus den Haushalten mit Zentralheizung fehlten 1,8 Tage weniger in der Schule und mussten wegen ihres Asthmas seltener zum Arzt oder in die Apotheke als die Kinder der Kontrollgruppe. Die Eltern berichteten nach Einbau der neuen Anlage seltener von einem schlechten Gesundheitszustand ihrer Kinder, Schlafstörungen durch Giemen oder trockenen Husten in der Nacht waren vermindert.

Fazit: Eine moderne Zentralheizung mit geringer Stickoxidbelastung in Wohn- und Schlafräumen kann zwar die Lungenfunktion von Kindern mit Asthma bronchiale nicht bessern, wohl aber die Symptome deutlich reduzieren.

Howden-Chapman P et al. Effects of improved home heating on asthma in community dwelling children: randomised controlled trial. BMJ 2008; 337: a1411

oder Rufen des Arztes bereits zu Beginn einer sich verschlechternden Symptomatik hat möglicherweise einige bedrohliche Notfallsituationen verhindert.

Eine Analyse der unterschiedlichen Schulungsmaßnahmen ergab bessere Ergebnisse für Programme mit mehr Einzelsitzungen und mehr Angeboten für interaktives Lernen.

Fazit: Asthmaschulungsprogramme reduzieren im Vergleich zu einer üblichen Betreuung die Zahl von Krankenhauseinweisungen und Notfallaufnahmen sowie das Risiko für eine Notfallaufnahme, nicht aber das relative Risiko für Krankenhauseinweisungen oder dringende asthmabedingte Arztbesuche. $\quad b k$

Coffman JM et al. Effects of asthma education on children's use of acute care services: a meta-analysis. Pediatrics 2008; 121: $575-86$ 